(C) Elsevier Sequoia S.A., Lausanne - Printed in Switzerland

\title{
TEMPERATURE EFFECTS ON FLUORESCENCE QUANTUM EFFICIENCY OF 9,10-DIPHENYLANTHRACENE
}

\author{
L. C. PEREIRA, 1. C. FERREIrA and MARILIA P. F. THOMAZ ${ }^{\dagger}$ \\ University of Minho, Molecular Physics Group, Braga (Portugal) \\ (Received January 27, 1978)
}

\section{Summary}

The fluorescence quantum efficiency of 9,10-diphenylanthracene in ethanol was measured with very dilute solutions ( between 170 and $350 \mathrm{~K}$, relative to solutions at room temperature. The results are compatible with a quantum efficiency of 0.88 at room temperature, which increases to 1.00 for temperatures below $190 \mathrm{~K}$.

\section{Introduction}

The value of the fluorescence quantum yield $\phi_{\mathrm{FM}}$ of 9,10 -diphenylanthracene (DPA) solutions has been a matter of controversy in the literature $[1,2]$ for some time, mainly because DPA has been used as a fluorescence standard [3]. Variation of $\phi_{F M}$ with temperature is in general assumed to be negligible but the effect of concentration on the fluorescence intensity of this molecule is still being questioned [4].

In order to help clarify this situation we have studied the variation of $\phi_{\mathrm{FM}}$ of very dilute solutions of DPA in ethanol (not more than $5 \times 10^{-6} \mathrm{M}$ ) as a function of temperature over a large temperature range (173 - $353 \mathrm{~K})$. Several factors which influence the fluorescence intensity have been investigated in order to obtain correct quantum yields. The precision in the measurement of relative fluorescence yields depends on a number of experimental conditions which have been discussed in the literature [4 - 6]. To avoid various sources of error Ware and Rothman [6] used an integrated sphere fluorimeter. While this type of experimental apparatus seems to be ideal in many respects it is desirable to analyse the experimental factors involved with conventional fluorimeters which can offer great versatility for many luminescence studies.

\footnotetext{
${ }^{\dagger}$ Present address: University of Aveiro, Physics Department, Portugal.
} 


\section{Experimental}

The ethanol and DPA samples were of high purity grades and were purified further by standard methods. Fluorescence spectroscopy failed to detect any impurity in the materials used.

Fluorescence measurements were carried out in a spectrofluorimeter which has been described previously [7] and for which a spectral correction curve was determined with a calibrated tungsten lamp and suitable quantum counters [8]. The excitation wavelength $\lambda_{\text {exe }}$ was kept at $376 \mathrm{~nm}$ with $2-3$ nm slits and the exciting light was cut off between measurements. The reflectivity of the optical system was measured as a function of temperature by reflecting the exciting light with a magnesium oxide screen placed in the Dewar; no variation was detected to within $1 \%$.

Polarization effects due to anisotropic photoselection [9] were negligible. The effect was investigated by passing the emission signal through a UV polarizer at parallel and perpendicular orientations. The ratio $I_{\eta} / I_{\perp}$ was shown to be constant within the range of temperatures under consideration and therefore was assumed to arise only from eigenpolarization of the apparatus [10].

Fluorescence spectra were obtained below room temperature using a cryostat unit similar to that described by Fisher [11] . A specially designed Dewar with three Spectrosil windows which allowed perpendicular reflection and transmission viewing was used. A continuous stream of dry nitrogen was blown onto the windows of the Dewar to avoid condensation at lower temperatures. For measurements above room temperature an insulated heating block was used. The temperature of the samples was monitored with a thermometer connected to a recorder, and the measurements were taken after the temperature had stabilized. Fluorescence spectra were recorded during both the cooling and the heating processes and a good reporducibility (to within $5 \%$ ) was achieved. The solutions were contained in a square cell which was sealed after degassing by the freeze-thaw technique. The variation of the optical density of the solutions with temperature was monitored by measuring the difference between the amount of light of wavelength $\lambda_{\text {exc }}$ transmitted by the solvent and by the sample as a function of temperature, with the same experimental conditions as for the fluorescence measurements. This method is able to detect whether the solvent remains transparent over the whole temperature range used. Lack of transparency due to small particles of ice or other impurities can result in diffuse reflection giving rise to an anomalous luminescence intensity [7].

\section{Results and discussion}

The variation of the fluorescence quantum yield with temperature was measured relative to its value at room temperature. The fluorescence spectra were recorded at various temperatures and the areas under the emission 
spectra were measured by the cutting and weighing method and were corrected for optical density variation.

The variation of the refractive index with temperature at an average wavenumber of $23250 \mathrm{~cm}^{-1}$ was calculated using the Lorenz-Lorentz expression [12]. Using an $n^{2}$ correction [5] we have calculated the values of $\left(\phi_{F M}\right)_{t_{1}}$ at a given temperature $t_{1}$ relative to the value $\left(\phi_{\mathrm{FM}}\right)_{t_{\mathrm{o}}}$ at room temperature $t_{0}$ using the expression

$$
\frac{\left(\phi_{F M}\right)_{t_{1}}}{\left(\phi_{F M}\right)_{t_{0}}}=\frac{\left(A_{F}\right)_{t_{2}}^{c}}{\left(A_{F}\right)_{t_{0}}^{c}} \frac{\left(n^{2}\right)_{t_{1}}}{\left(n^{2}\right)_{t_{0}}}
$$

where $\left(A_{\mathrm{F}}\right)_{t}^{\mathrm{c}}$ is the area obtained at temperature $t$ after correction for optical density variation. The oscillator strength of the solution is assumed to be independent of temperature within the range considered [13].

The results obtained are plotted in Fig. 1 by normalizing to $\phi_{\mathbf{F M}}=1.0$ in the lower temperature region, assuming that $\phi_{\mathrm{FM}}$ tends to unity in this region. As shown in Fig. 1 a slight variation with temperature is found with $\phi_{\text {FM }}=0.88$ at room temperature. This behaviour should be compared with the results reported by Heinrich and coworkers [14] who have quoted $\phi_{\mathrm{FM}}=$ 0.94 at room temperature and $\phi_{\mathrm{FM}}=1.0$ at $77 \mathrm{~K}$ using $10^{-5} \mathrm{M}$ solutions of DPA in ethanol. In contrast, Mantulin and Huber [15] have reported no variation of $\phi_{\mathrm{FM}}$ with temperature using ethanol-isopentane-alcohol as solvent but the concentration used was not given. A straightforward $n^{2}$ correction was questioned by Morris et al. [16] who pointed out that the correction is a function of the sample compartment and the viewing angle. According to the calculations of Lumb [17] an $n^{2}$ correction should be applied for the excitation and emission geometry conditions used in this work.

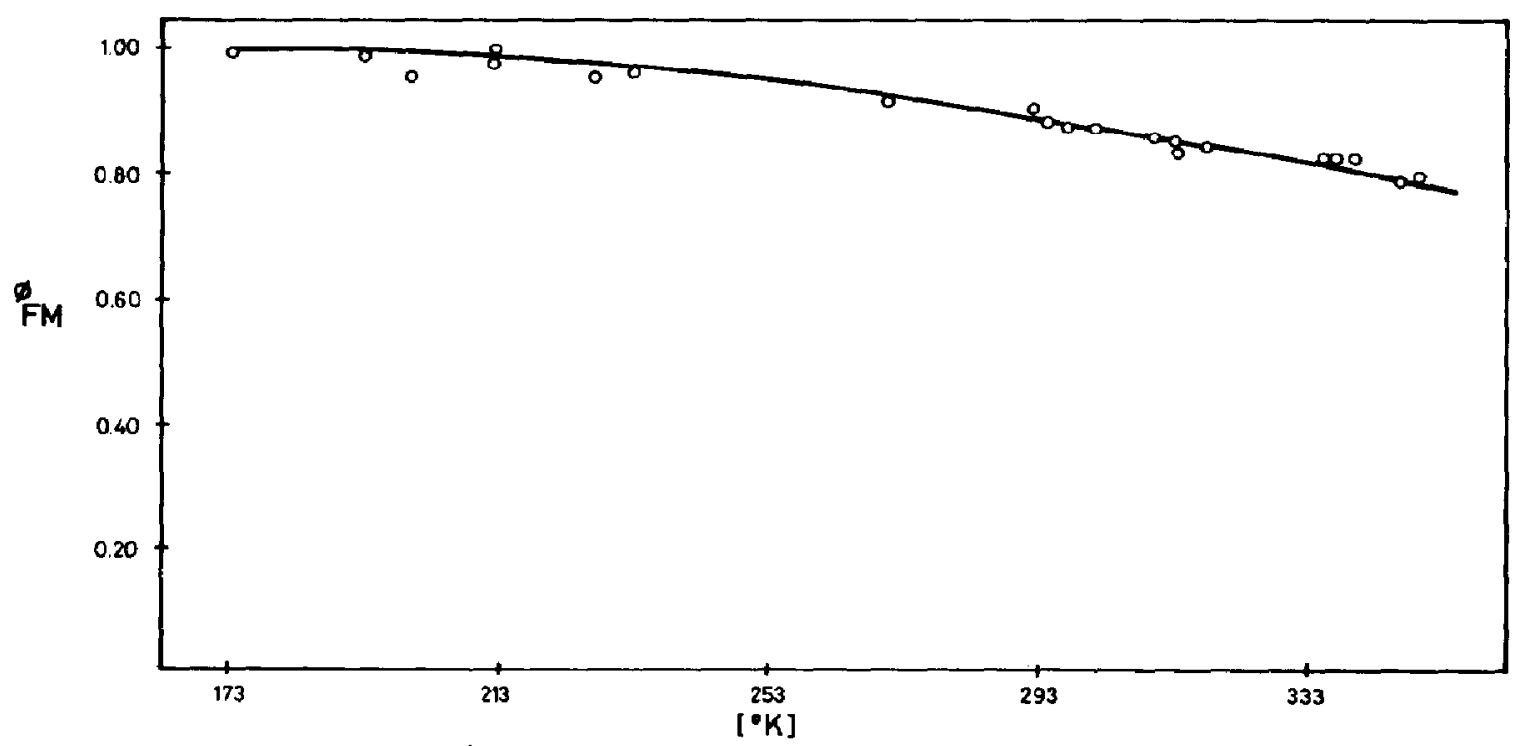

Fig. 1. Fluorescence quantum yields of $5 \times 10^{-6} \mathrm{M}$ solutions of DPA in ethanol as a function of temperature. 
A decrease of $\phi_{\mathrm{FM}}$ with temperature is normal for aromatic molecules and could be interpreted in this case as being due to a slight increase of the triplet yield $\phi_{\text {TM }}$ with temperature. It is interesting to note that Stevens and Mills [18] have reported $\phi_{\mathrm{TM}}=\mathbf{0 . 1 3}$ for this molecule at room temperature using a photoperoxidation method and have suggested the possibility of the existence of a second triplet below the first singlet excited state $\mathbf{S}_{1}$. Parker and Joyce [19] reported $\phi_{\mathrm{FM}}=0.89$ and $\phi_{\mathrm{TM}}=0.03$ for $5 \times 10^{-5} \mathrm{M}$ solutions in ethanol in which P-type delayed fluorescence was also measured.

Our results can also be compared with those of Ferguson and Mau [20] who found that $\phi_{\mathrm{FM}}$ had a value of 0.90 in ethanol solutions at room temperature and approached unity as the temperature was lowered. Using the same solvent, a value of $\phi_{F M}$ of 0.95 was reported by Huber and coworkers $[15,21]$. More evidence has been produced indicating that, at least in some solvents, $\phi_{\text {FM }}$ of DPA is not unity at room temperature $[4,22]$. For example Cehelnik et al. [23] obtained $\phi_{\mathrm{FM}}=1.00$ for all-trans-1,6 diphenyl-1,3,5hexatriene in 3-methylpentane below $165 \mathrm{~K}$ relative to a value of 0.83 for DPA in cyclohexane at room temperature.

Some experimental problems involved in the measurement of relative fluorescence yields are still difficult to assess. The use of techniques such as the integrating sphere and calorimetric determination for measurement of fluorescence yields is desirable because they avoid problems due to reabsorption effects and refractive index corrections. Ware and Rothman [6], using an integrating sphere fluorimeter, have reported that $\phi_{\mathrm{FM}}=1.00$ to within 5\% for DPA in cyclohexane using quinine bisulphate in $1 \mathrm{~N} \mathrm{H}_{2} \mathrm{SO}_{4}$ as the primary standard. However, Mardelli and Olmsted [24], using a calorimetric technique, have found $\phi_{\mathrm{FM}}=0.88$ for DPA in ethanol in agreement with the results reported in this work. Solvent effects on the fluorescence yield of th is molecule as found by other authors $[14,16,24,25]$ may explain the discrepancy between these results and those of Ware and Rothman [6] .

In view of the results indicated it can be concluded that DPA is not an ideal fluorescence standard, at least at room temperature, mainly due to solvent effects, self-absorption and secondary fluorescence. However, these results and others $[14,26]$ support the view that $\phi_{F M}=1.00$ at low temperatures, suggesting that DPA is a convenient standard for phosphorescence yield measurements at $77 \mathrm{~K}$.

\section{Acknowledgment}

The authors gratefully acknowledge partial financial support from a NATO grant.

\section{References}

1 J. B. Birks, Photophysics of Aromatic Molecules, Interscience, New York, 1970, p. 103.

2 I. B. Berlman, Chem. Phys, Lett., 21 (1973) 344. 
3 I. B. Berlman, Handbook of Fluorescence Spectra of Aromatic Molecules (2nd edn.), Academic Press, New York, 1971.

4 J. B. Birks, J. Lumin., 9 (1974) 31; J. Res. Nat. Bur. Stand., Sect. A, 80 (1976) 389.

5 J. N. Demas and G. A. Crosby, J. Phys. Chem., 75 (1971) 991.

6 W. R. Ware and W. Rothman, Chem. Phys. Lett., 39 (1976) 449.

7 M. D. Lumb, C. Lloyd Braga and L. C. Pereira, Trans. Faraday Soc., 65 (1969) 1992.

8 M. D. Lumb, M. I. Barradas, L. C. Pereira and C. Lloyd Braga, Rev. Fis. Quim. Eng., Ser. A, 1 (1969) 1.

9 E. D. Cehelnik, K. D. Mielenz and R. A. Velapoldi, J. Res. Nat. Bur. Stand., Sect. A, 79 (1975) 1.

10 M. Almgren, Photochem. Photobiol., 8 (1968) 281.

11 Y. Hirshberg and E. Fischer, Rev. Sci. Instrum., 30 (1959) 197.

12 L. C. Pereira, Ph.D. Thesis, Univ. Nottingham, 1972.

13 U. P. Klochkov and S. M. Korotkov, Opt. Spectrosk., Suppl. 2 (1966) 11 (English transl.).

14 G. Heinrich, S. Schoof and H. Gusten, J. Photochem., $3(1974 / 75) 315$.

15 W. W. Mantulin and J. R. Huber, Photochem. Photobiol., 17 (1973) 139.

16 J. V. Morris, M. A. Mahaney and J. R. Huber, J. Phys. Chem., 80 (1976) 969.

17 M. D. Lumb, Rev. Fis. Quim. Eng., Ser. A, 1 (1969) 27.

18 B. Stevens and L. E. Mills, Chem. Phys. Lett., 15 (1972) 381.

19 C. A. Parker and T. A. Joyce, Chem. Commun., (1967) 744.

20 J. Ferguson and A. W. H. Mau, Chem. Phys. Lett., 14 (1972) 245.

21 J. R. Huber, M. A. Mahaney and W. W. Mantulin, J. Photochem., $2(1973 / 74) 67$.

22 P. Lentz, H. Blume and D. Schulte-Frohlinde, Ber. Bunsenges. Phys. Chem., 74 (1970) 484 .

23 E. D. Cehelnik, R. B. Cundall, J. R. Lockwood and T. F. Palmer, J. Phys. Chem., 79 (1975) 1369.

24 M. Mardelli and J. Olmsted III, J. Photochem., 7 (1977) 277.

25 D. J. S. Birch and R. E. Imhof, Chem. Phys. Lett., 32 (1975) 56.

26 E. C. Lim, J. D. Laposa and J. M. H. Yu, J. Mol. Spectrose., 19 (1966) 412. 